\title{
Unilateral and Reversible Hypoglossal Nerve Palsy Due to Infectious Mononucleosis
}

\author{
Ji-Hoon Na, MD' ${ }^{1}$ Ji Hyen Lee, $\mathrm{MD}^{2}$ \\ 'Department of Pediatrics, Gangnam Severance Hospital, Yonsei University College of Medicine, Seoul, Korea \\ ${ }^{2}$ Department of Pediatrics, Ewha Womans University School of Medicine, Seoul, Korea
}

Received: July 13, 2021

Revised: July 28, 2021

Accepted: August 1, 2021

\section{Corresponding author:}

Ji Hyen Lee, MD

Department of Pediatrics, Ewha

Womans University School of

Medicine, 260 Gonghang-daero,

Gangseo-gu, Seoul 07804, Korea

Tel: +82-2-6986-1766

Fax: +82-2-6986-3024

E-mail: leejihyen@ewha.ac.kr
Infectious mononucleosis is a benign, self-limiting disorder caused by primary Epstein-Barr virus (EBV) infection. Various neurological diseases, such as encephalitis, meningitis, acute inflammatory polyneuropathy, Bell's palsy, and peripheral neuropathy, may be associated with EBV infection; however, involvement of the central nervous system (CNS) is relatively uncommon [15]. Rare cases of hypoglossal nerve palsy associated with acute $\mathrm{EBV}$ infection have been reported in children [6-9]. Herein, we report a case of unilateral and reversible hypoglossal nerve palsy with acute EBV infection. The study was reviewed and approved by the Ewha Womans University Seoul Hospital Institutional Review Board (IRB File No: SEUMC 2021-07-028). The requirement for informed consent was waived by the board.

A previously healthy 17-year-old man presented with a 7-day history of severe left sublingual pain, paralysis, and lingual dysarthria. He had not received any dental treatment in recent years. However, he had recently experienced upper respiratory tract infection symptoms, which included cough and rhinorrhea, for 5 days before he was left with sublingual paralysis. He was barely able to move his tongue to the left side, with severe pain in the tongue that disturbed his sleep and made it difficult to swallow food
(Fig. 1). He complained of occasional but severe pain extending to the tongue, cheeks, throat, and chest, with difficulty breathing. The patient's vital signs were stable and within the normal range, and his body temperature was normal 1 day before arrival at the hospital. Blood tests revealed mild leukocytosis and elevation of C-reactive protein, with no electrolyte abnormalities except mild hyponatremia. On physical examination, multiple lymph nodes were palpable, including level II left cervical lymphadenopathy, with the largest node measuring approximately $1.0 \times 1.5$ $\mathrm{cm}$. The lymph nodes were firm, discrete, and minimally tender. No enlargement of the liver or spleen and no skin rashes were observed. Magnetic resonance (MR) imaging of the brain and neck after hospitalization demonstrated level II bilateral reactive lymphadenopathy, while MR angiography showed no specific findings. Neck computed tomography showed levels II and III bilateral reactive lymphadenopathy and enlarged adenoids and palatine tonsils, with bilateral tonsilloliths. In serologic tests for viruses, herpes simplex virus, varicella-zoster, and mumps were not detected. However, EBV serology results were positive for EBV viral capsid antigen (VCA) immunoglobulin M (IgM), EBV VCA $\mathrm{IgG}$, early antigen IgG, and Epstein-Barr nuclear antigen IgG; furthermore, negative findings on

Copyright (C) 2022 Korean Child Neurology Society

This is an Open Access article distributed under the terms of the Creative Commons Attribution Non-Commercial License (http://creativecommons.org/licenses/by-nc/4.0/) which permits unrestricted non-commercial use, distribution, and reproduction in any medium, provided the original work is properly cited. 
EBV polymerase chain reaction testing confirmed the reactivation of EBV. The patient was administered intravenous (IV) steroids and gabapentin. The pain was effectively controlled using gabapentin. After administering IV dexamethasone for 5 days, oral prednisolone was prescribed. The patient fully recovered, and no other sequelae remained (Fig. 1).

EBV may affect many organ systems; however, CNS involvement of EBV is uncommon and not fully understood [1]. CNS manifestations may include aseptic meningitis, encephalitis, myelitis, optic neuritis, cranial nerve palsies, transverse myelitis, "Alice in Wonderland" syndrome, and Guillain-Barre syndrome. Recently, three pathogenetic pathways underlying the CNS manifestations of EBV have been identified: first, direct invasion of EBV into nerves, second, immune-mediated reaction in the nervous system due to EBV, and third, reactivation of latent infection [1]. Although isolated cranial nerve palsies occurring in the setting of acute EBV infection have been reported for almost all 12 cranial nerves, there are limited reports of hypoglossal nerve palsy due to EBV infection reactivation in adolescents.

Acute EBV infections account for approximately $2 \%$ to $5 \%$ of infection-related neurological diseases [2,3]. Hausler et al. [4] investigated 48 pediatric patients with neurological complications of acute and persistent EBV infection and reported a similar (4\%; $2 / 48$ ) rate of infection-related neurological disease. Reactivated or chronic EBV infections were found in 17\% (8/48) of patients. Furthermore, considering that these infections were encountered in only a few case reports described in the literature, Hausler et al. [4] suggested that the impact of EBV reactivation on pediatric neurological disease may be higher than commonly assumed. In rare cases, reactivated EBV infections may cause persistent neurologic sequelae, such as hippocampal lesions.

Hypoglossal nerve palsy may result from a variety of conditions, including neoplasms, trauma, aneurysms, and infections. Keane [5] reviewed 100 patients with 12 th nerve palsy observed at a single hospital over 26 years. In his study, hypoglossal nerve palsy due to infection was infrequently identified, in just 4\% (4/100) of patients, and only $15 \%$ of cases had a complete or nearly complete recovery. Hypoglossal palsy associated with EBV infection, unlike that caused by many other factors, is characteristically isolated, unilateral, and reversible [6-9]. If hypoglossal nerve palsy is either bilateral or associated with other cranial nerve signs, neuroimaging is mandatory to exclude other structural causes. Early EBV infection status should be evaluated in cases of isolated unilateral hypoglossal palsy. Furthermore, detection of serologic signs of EBV infection is of prognostic significance, and may predict recovery within 6 months. Four previously reported cases showed recovery within 6 months, two of which were treated using corticosteroids. These
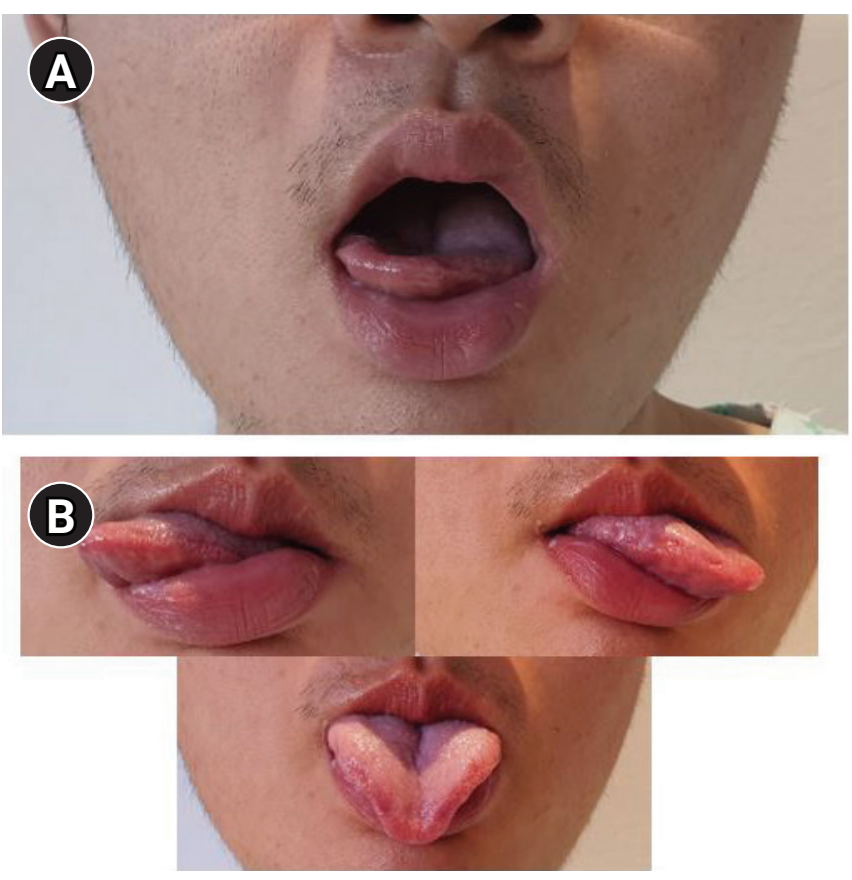

Fig. 1. The status of the patient's tongue movement before and after treatment. (A) Photograph of the patient's tongue showing deviation to the right side, indicating left side hypoglossal palsy. The patient had difficulty pushing his tongue forward due to excruciating pain and was unable to move his tongue to the left side. (B) Improvement of tongue movement after treatment permitting its free movement in all directions (post-treatment).

cases of CNS involvement of EBV had the same clinical picture as ours $[7,8]$.

Herein, we report a case of unilateral and reversible hypoglossal nerve palsy resulting from the reactivation of infectious mononucleosis. Although cases of hypoglossal nerve palsy after infection with EBV are rare and cause severe symptoms, such cases could be managed with symptomatic treatments such as steroids and pain control medications, which have an excellent prognosis, as observed in our case. Future follow-up is required to determine whether recurrent neurological symptoms due to reactivation of EBV occur in this patient.

\section{Conflicts of interest}

No potential conflict of interest relevant to this article was reported.

\section{ORCID}

Ji-Hoon Na, https://orcid.org/0000-0002-3051-2010

Ji Hyen Lee, https://orcid.org/0000-0002-2234-1055 


\section{Author contribution}

Conceptualization: JHN and JHL. Data curation: JHN. Formal analysis: JHN. Methodology: JHN. Project administration: JHN. Visualization: JHN and JHL. Writing-original draft: JHN and JHL. Writing-review \& editing: JHN and JHL.

\section{References}

1. Cheng H, Chen D, Peng X, Wu P, Jiang L, Hu Y. Clinical characteristics of Epstein-Barr virus infection in the pediatric nervous system. BMC Infect Dis 2020;20:886.

2. Yabuki S, Kazahaya Y, Kubonishi I. Epstein-Barr virus antibodies in neurological diseases. Folia Psychiatr Neurol Jpn 1985;39: 85-93.

3. Bale JF Jr. Human herpesviruses and neurological disorders of childhood. Semin Pediatr Neurol 1999;6:278-87.

4. Hausler M, Ramaekers VT, Doenges M, Schweizer K, Ritter K, Schaade L. Neurological complications of acute and persistent Epstein-Barr virus infection in paediatric patients. J Med Virol 2002;68:253-63.

5. Keane JR. Twelfth-nerve palsy. Analysis of 100 cases. Arch Neurol 1996;53:561-6.

6. Chatterjee AK. An unusual neurological complication of infectious mononucleosis. Br J Clin Pract 1961;15:595-6.

7. Sibert JR. Hypoglossal nerve palsy complicating a case of infectious mononucleosis. Postgrad Med J 1972;48:691-2.

8. DeSimone PA, Snyder D. Hypoglossal nerve palsy in infectious mononucleosis. Neurology 1978;28:844-7.

9. Wright GD, Lee KD. An isolated right hypoglossal nerve palsy in association with infectious mononucleosis. Postgrad Med J 1980;56:185-6. 